\title{
Synthesis of Heteroatom $\mathrm{Rh}-\mathrm{ReO}_{x}$ Atomically Dispersed Species on $\mathrm{Al}_{2} \mathrm{O}_{3}$ and Their Tunable Catalytic Reactivity in Ethylene Hydroformylation
}

\author{
Mingjie $\mathrm{Xu}^{1}$, Insoo $\mathrm{Ro}^{2}$, George Graham ${ }^{3}$, Xiaoqing $\mathrm{Pan}^{4}$ and Phillip Christopher ${ }^{2}$
}

${ }^{1}$ UCI, United States, ${ }^{2}$ UCSB, United States, ${ }^{3}$ UM, United States, ${ }^{4}$ Department of Physics and Astronomy, University of California, Irvine, CA 92697, Irvine, California, United States

Atomically dispersed late-transition-metal catalysts exhibit distinct catalytic reactivity and selectivity compared to metal clusters in many reactions. Realizing the potential benefits of these catalysts requires active site uniformity and control of their local environment. Here, we propose a catalyst synthesis route for manipulating the local environment of atomically dispersed metal-active sites. This was achieved via the targeted deposition of Rh precursors near atomically dispersed $\operatorname{ReOx}$ on $\gamma$ A12O3 using electrostatic interactions. CO probe molecule infrared spectroscopy and aberration-corrected scanning transmission electron microscopy suggested that Rh could be preferentially located near a single ReOx species or multiple $\mathrm{ReOx}$ species by controlling $\mathrm{ReOx}$ loading. $\mathrm{Rh}-\mathrm{ReOx}$ interactions promoted catalytic reactivity and selectivity for ethylene hydroformylation. Kinetic measurements suggested that ReOx species withdrew charge from $\mathrm{Rh}$, weakening $\mathrm{Rh}-\mathrm{CO}$ interactions, which promoted the concentration of vacant sites under reaction conditions and in turn catalytic reactivity. This work demonstrates a general synthetic approach for creating atomically dispersed heteroatom species consisting of oxophilic and late-transition metals and the importance of regulating the local environment of atomically dispersed metals for maximizing catalytic performance.

High-angle annular dark field scanning transmission electron microscopy (HAADF-STEM) was performed on a JEOL Grand ARM300CF microscope at $300 \mathrm{kV}$. The HAADF-STEM images of Rh/ReOx-Al2O3 sample enables the direct observation of co-localized atomically dispersed $\mathrm{Rh}$ and $\mathrm{Re}$ species distinguished by $\mathrm{Z}$ contrast (Figure 1).

In conclusion, we demonstrated a synthetic approach for tuning the local environment of atomically dispersed Rh species through the targeted deposition of Rh near atomically dispersed ReOx species using electrostatic interactions. The charge states of dispersed Rh species were systematically controlled by varying the local coverage of $\mathrm{ReOx}$ around Rh. Correlations between the $\mathrm{CO}$ stretching frequency of $\mathrm{Rh}(\mathrm{CO}) 2$ species and the catalytic properties indicate that the charge state of Rh strongly influenced the reactivity and selectivity in ethylene hydroformylation. Catalytic activity was promoted via interactions between dispersed $\mathrm{Rh}-\mathrm{ReOx}$ species ( $\mathrm{ReOx}$ withdrawing charge from $\mathrm{Rh}$ ), rendering a higher concentration of vacant sites because of a decrease in the $\mathrm{CO}$ binding energy. This work demonstrates how the local environment of atomically dispersed late transition metals can be systematically controlled to tune catalytic reactivity. The synthetic protocols employed here should be generally useful for the systematic engineering of the local environment of atomically dispersed metal species and could be employed to probe electronic structure-reactivity relationships.

\section{Acknowledgement}

We acknowledge funding support from the University of California-Santa Barbara (UCSB). P.C. acknowledges partial support from the National Science Foundation (NSF) CAREER grant number CBET-1823189. Use of the ICP and BET equipment in 
the UCSB MRL Shared Experimental Facilities is acknowledged, which are supported by the MRSEC Program of the National Science Foundation under award no. DMR 1720256. STEM experiments were conducted using the facilities in the Irvine Materials Research Institute at the University of California-Irvine (UCI). The work at UCI was supported by the National Science Foundation through the grant number DMR-1506535.
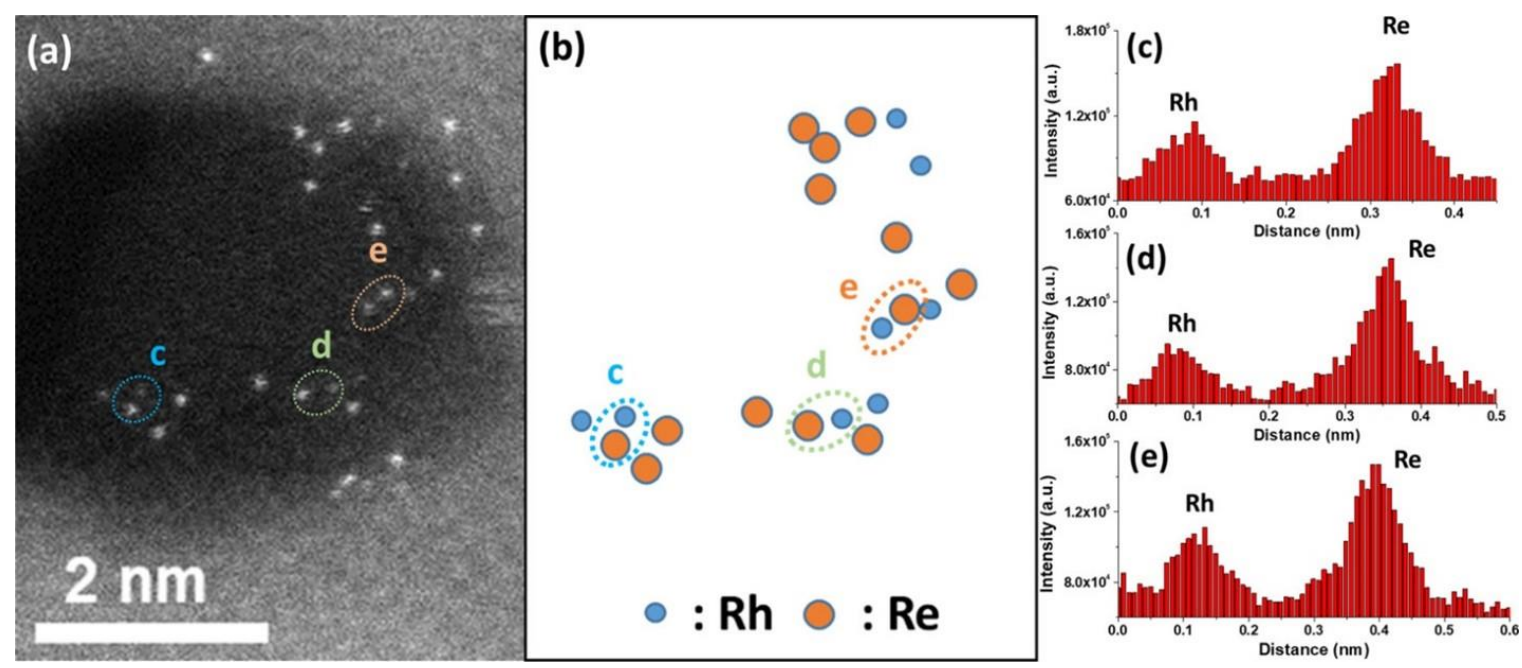

Figure 1. Figure 1 (a) HAADF-STEM image of $\mathrm{Rh} / 1 \mathrm{ReOx}-\mathrm{Al} 2 \mathrm{O} 3$ after ex situ calcination at $623 \mathrm{~K}$. (b) Using the different scattering intensities of Rh and Re, a schematic was created identifying Rh and Re species in (a). (c-e) Corresponding line scan intensity analysis of the three selected dimers shown in $(a, b)$.

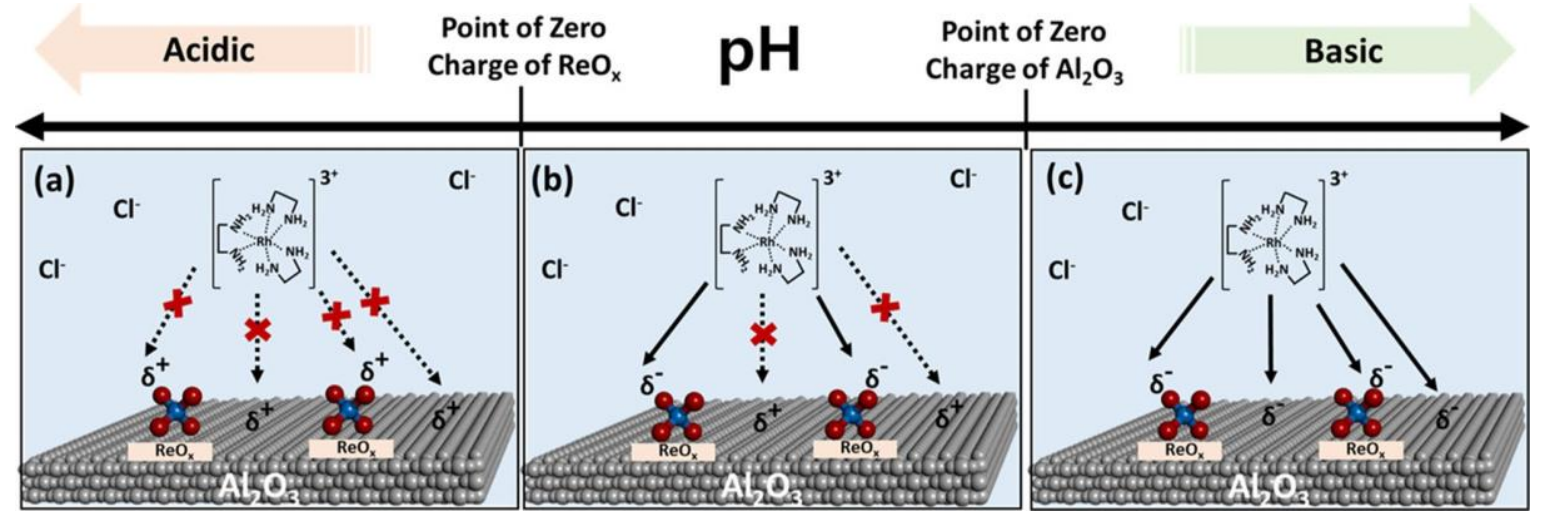

Figure 2. Schematic of the SEA-based synthetic protocol allowing selective deposition of the cationic Rh precursor $([(\mathrm{H} 2 \mathrm{NCH} 2 \mathrm{CH} 2 \mathrm{NH} 2) 3 \mathrm{Rh}] 3+)$ near ReOx domains via electrostatic attraction between metal oxide surfaces and charged precursors in solution at three different $\mathrm{pH}$ regions. The charge state shown in the figure represents a net charge of $\mathrm{ReOx}$ and the $\mathrm{Al} 2 \mathrm{O} 3$ surface after protonation/deprotonation depending on the $\mathrm{pH}$. (a) At a pH below the $\mathrm{PZC}$ of $\mathrm{ReOx}$ and $\mathrm{Al} 2 \mathrm{O} 3$, the positively charged $\mathrm{ReOx}$ and $\mathrm{Al} 2 \mathrm{O} 3$ surface after protonation do not interact with the cationic Rh precursor, resulting in negligible $\mathrm{Rh}$ deposition on the metal oxide surface. (b) At a pH between the PZC of $\mathrm{ReOx}$ and Al2O3, only hydroxyl groups on $\mathrm{ReOx}$ are deprotonated and create negatively charged oxygen species, leading to a potential for selective deposition of Rh precursor near negatively charged $\mathrm{ReOx}$. (c) At a pH above the PZC of $\mathrm{ReOx}$ and $\mathrm{Al} 2 \mathrm{O} 3$, hydroxyl groups on the $\mathrm{ReOx}$ and $\mathrm{Al} 2 \mathrm{O} 3$ surface are deprotonated, creating negatively charged oxygen species that both induce electrostatic interactions with cationic Rh precursor.

\section{References}

Ro, I., Xu, M., Graham, G. W., Pan, X. \& Christopher, P. Synthesis of Heteroatom Rh-ReOx Atomically Dispersed Species on Al2O3 and Their Tunable Catalytic Reactivity in Ethylene Hydroformylation. ACS Catalysis 9, 10899-10912, doi:10.1021/acscatal.9b02111 (2019) 